\title{
Novel features of quantum conduction in a constriction
}

\author{
E. Tekman and S. Ciraci \\ Department of Physics, Bilkent University, Bilkent 06533, Ankara, Turkey
}

(Received 23 November 1988)

\begin{abstract}
The effects of the geometry and temperature on the quantum conductance for one-dimensional (1D) ballistic transport through a constriction in a $2 \mathrm{D}$ electron gas are investigated by use of a refined formalism. As the length of the constriction increases, weak oscillations around the classical conductance evolve into a steplike structure and the resonances on the plateaus become pronounced. Quantization at integer multiples of $2 e^{2} / h$ occurs only for uniform constriction of finite length. At finite temperature of $\sim 0.6 \mathrm{~K}$ significantly long uniform constriction is necessary to observe plateaus devoid of resonance structure.
\end{abstract}

The ballistic transport of the electron gas in lower dimension has become the subject of current interest. ${ }^{1-3}$ Recently, van Wees et al. ${ }^{4}$ and Wharam et al. ${ }^{5}$ achieved the measurement of the conductance $G$, through a narrow constriction between two reservoirs of two-dimensional electron gas (2D EG) in high-mobility GaAs-GaAlAs heterostructure. The constriction they made by a split gate was significantly narrow so that its width $w$ was comparable with the Fermi wavelength $\left(w \sim \lambda_{F}\right)$, and also significantly short $\left(d<l_{e}\right.$ electron mean free path) so that electrons can move ballistically. The resulting conductance of the transport through this constriction was found to change with $w$ (or gate voltage) in quantized steps of approximately $2 e^{2} / h$. Theories ${ }^{4,5}$ based on the quantization of the transverse momentum have had only limited success so far to explain this quantum resistance (or conductance). Although the sharp, quantized steps have been predicted in terms of a new channel opened by a subband dipping into the Fermi level, an elaborate theory is required to reveal the crucial features of this important observation. $^{4,5}$ In fact, it is not clear yet how the details of the quantum conductance depend on temperature and the width and length of the constriction, ${ }^{6-8}$ and how the

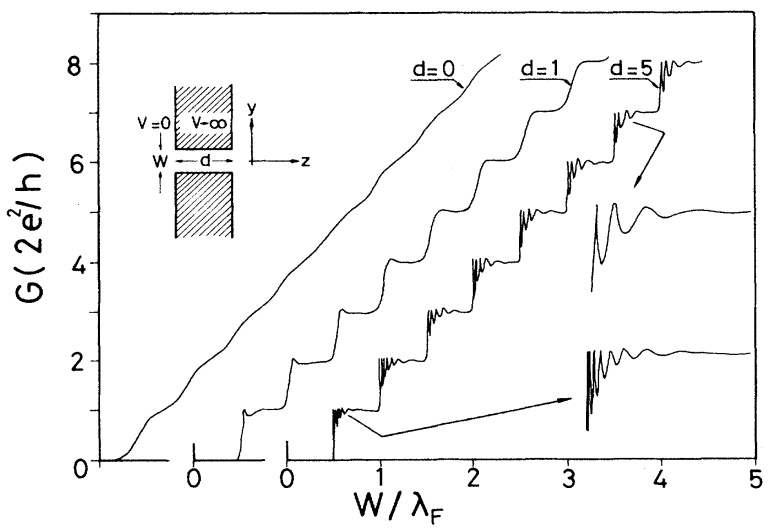

FIG. 1. Quantum conductance $G$ of a uniform constriction calculated for various lengths $d$. The unit of length is $\lambda_{F}$, and $T=0 \mathrm{~K}$. The resonance structure of the first and seventh channels are magnified by the inset. geometry of the contact affects the behavior of $G$.

In this paper we present a thorough analysis of the quantum conductance through a constriction by using a refined formalism. The expression we derived provides an exact calculation of $G$ and allows the investigation of the effects of temperature and contact geometry. A number of important results emerging from the present study are as follows: (i) For an abrupt and uniform constriction (see the inset in Fig. 1), the quantization of $G$ at $T=0 \mathrm{~K}$ occurs at finite $d$, but quantum effects become weaker as $d$ is shortened. A resonance structure superimposed on the plateaus becomes pronounced as $d$ increases. The number of resonances on a plateau decreases with increasing $w$, but increases with increasing $d$. (ii) For a wedgelike constriction (see the inset in Fig. 2), the step structure becomes significant only for large wedge angle $\alpha$. (iii) At a typical experimental temperature $(T \lesssim 0.6 \mathrm{~K})$ the resonance structure disappears only for large $d\left(d>5 \lambda_{F}\right)$. For even higher temperatures $(T \sim 5 \mathrm{~K})$ the steplike structure is smoothed, but is still recognizable.

To formulate the conductance in a finite and abrupt constriction we first consider the system consisting of semi-infinite constriction of a uniform width $w$ for $z \leq 0$,

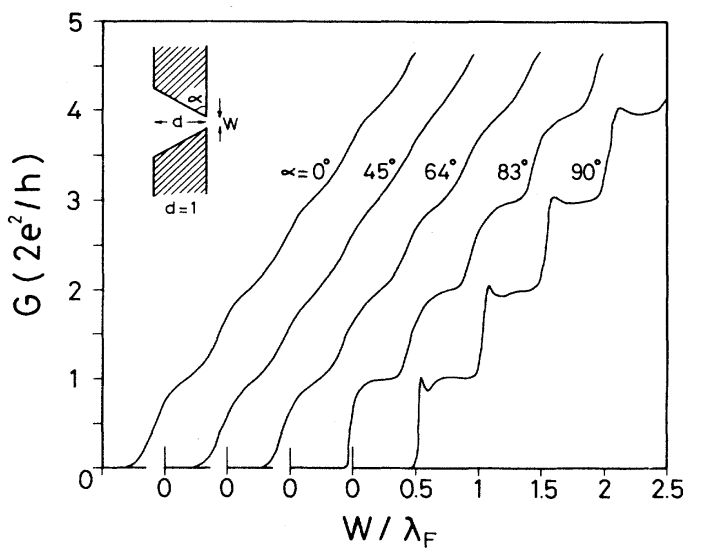

FIG. 2. Quantum conductance $G$ of a wedgelike constriction calculated for various wedge angles $\alpha$. The constriction length $d=1$ and $T=0 \mathrm{~K}$. 
and a 2D EG for $z \geq 0$. For an electron incident from the constriction side, the wave function of energy $E$ is written as

$$
\begin{aligned}
& \psi_{n}(y, z \leq 0)=e^{i \gamma_{n} z} \phi_{n}(y)+\sum_{m=1}^{\infty} e^{-i \gamma_{m} z} \phi_{m}(y) r_{m n} \\
& \psi_{n}(y, z \geq 0)=\int_{-\infty}^{\infty} d \kappa A_{n}(\kappa) e^{i k_{z}(\kappa) z} e^{i \kappa y}
\end{aligned}
$$

where $\gamma_{n}^{2}=2 m\left(E-\epsilon_{n}\right) / \hbar^{2}, k_{z}^{2}(\kappa)+\kappa^{2}=2 m E / \hbar^{2}$, and $\left[\phi_{n}, \epsilon_{n}\right]$ is the $n$th eigenstate of the infinite-well constriction potential. The reflection coefficient $r_{m n}$ and the transmission function $A_{n}(\kappa)$ are determined from the boundary conditions at $z=0$. By introducing the transversal Fourier transform

$$
\Phi_{n}(q)=(2 \pi)^{-1 / 2} \int_{-\infty}^{\infty} d y e^{-i q y} \phi_{n}(y)
$$

the continuity equations can be expressed in $q$ space; therefore $A_{n}(q)$ is eliminated to yield the relation

$$
\Sigma_{m}\left[\gamma_{m}+k_{z}(q)\right] \Phi_{m}(q) r_{m n}=\left[\gamma_{n}-k_{z}(q)\right] \Phi_{n}(q)
$$

This equation is multiplied by $\Phi_{j}^{*}(q)$ and integrated over q. The resulting equations can be cast in a matrix form:

$$
\tilde{\mathbf{r}}=(\tilde{\Gamma}+\tilde{\mathbf{K}})^{-1}(\tilde{\Gamma}-\tilde{\mathbf{K}}),
$$

where $\tilde{\Gamma}$ has the elements $\Gamma_{i j}=\gamma_{i} \delta_{i j} . \quad \gamma_{i}$ is either real or imaginary, and thus $\tilde{\Gamma}=\tilde{\Gamma}_{\mathbf{R}}+i \tilde{\Gamma}_{\mathbf{I}}, \tilde{\Gamma}_{\mathbf{R}}$ and $\tilde{\Gamma}_{\mathbf{I}}$ being diagonal matrices with positive real elements. $\tilde{\mathbf{K}}$ is given by $K_{i j}=\left\langle\Phi_{i}\left|k_{z}(q)\right| \Phi_{j}\right\rangle$. The expression for $\tilde{\mathbf{r}}$ is reminiscent of the reflection coefficient obtained for 1D step potential, $r=\left(k^{\prime}-k\right) /\left(k^{\prime}+k\right), k$ and $k^{\prime}$ denoting incident and transmitted wave vectors, respectively. To formulate the incidence from the 2D EG we consider the mirror image of the above geometry. Following similar steps we obtain the transmission coefficients corresponding to the incident wave vector $\mathbf{k}=\left(k_{0}, \kappa_{0}\right)$ :

$$
\tilde{\mathbf{t}}_{\mathbf{k}}=\sqrt{2 \pi}(\tilde{\boldsymbol{\Gamma}}+\tilde{\mathbf{K}})^{-1} 2 k_{0} \tilde{\boldsymbol{\Phi}}^{\dagger}
$$

Here the vectors, $\tilde{\mathbf{t}}_{\mathbf{k}}$ and $\tilde{\boldsymbol{\Phi}}$ have elements $t_{n \mathbf{k}}$ and $\Phi_{n}\left(\kappa_{0}\right)$, respectively. The equation for $\tilde{\mathbf{t}}_{\mathbf{k}}$ is analogous to the $1 \mathrm{D}$ semi-infinite barrier transmission coefficient $t=2 k$ / $\left(k+k^{\prime}\right)$.

Finally, to obtain the conductance for a constriction of finite length $d$ and width $w$ between two reservoirs of 2D EG as described in the inset of Fig. 1, we consider the incident wave $\mathbf{k}$. This gives rise to right-going constriction states with amplitudes $\tilde{\mathbf{t}}_{\mathbf{k}}$ which are reflected back at the right boundary with amplitudes $\tilde{\mathbf{r}}$ and the phase factors $e^{i \tilde{\Gamma} d}$. Then the wave function including multiple reflections in the constriction region can be written as

$$
\tilde{\boldsymbol{\Phi}}(y)\left[e^{i \tilde{\mathbf{\Gamma}} z} \tilde{\boldsymbol{\Theta}}(\mathbf{k})+e^{-i \tilde{\mathbf{\Gamma}} z} \tilde{\Delta}(\mathbf{k})\right],
$$

where $\tilde{\boldsymbol{\Theta}}(\mathbf{k})=\left[\tilde{\mathbf{I}}-\left(\tilde{\mathbf{r}} e^{i \tilde{\mathbf{\Gamma}} d}\right)^{2}\right]^{-1} \tilde{\mathbf{t}}_{\mathbf{k}}$ and $\tilde{\Delta}(\mathbf{k})=e^{i \tilde{\mathbf{\Gamma}} d} \tilde{\mathbf{r}} e^{i \tilde{\mathbf{\Gamma}} d}$ $\times \tilde{\boldsymbol{\theta}}(\mathbf{k})$. Next we evaluate the current operator for the state $\mathbf{k}$ and integrate over all the states at the Fermi level to obtain the conductance

$$
G=\frac{e^{2}}{\pi h} \int_{-k_{F}}^{k_{F}} \frac{d \kappa}{k_{z}(\kappa)}\left\{\left[\tilde{\boldsymbol{\Theta}}^{\dagger}(\mathbf{k}) \tilde{\Gamma}_{\mathrm{R}} \tilde{\boldsymbol{\Theta}}(\mathbf{k})-\tilde{\Delta}^{\dagger}(\mathbf{k}) \tilde{\Gamma}_{\mathrm{R}} \tilde{\Delta}(\mathbf{k})\right]+2 \operatorname{Im}\left[\tilde{\boldsymbol{\Theta}}^{\dagger}(\mathbf{k}) \tilde{\Gamma}_{\mathrm{I}} \tilde{\Delta}(\mathbf{k})\right]\right\}
$$

In this expression the first and second terms in the parentheses are related to the right-going and left-going states, respectively. The contribution of the evanescent states are expressed by the third term. At small $d$ the sharp rises in conductance corresponding to the opening of a new channel are smoothed out by the evanescent states. In contrast to methods ${ }^{4-8}$ proposed earlier, the contributions of various types of states are explicitly given in the present formalism. This provides a better description of the quantum phenomena taking place in the constriction, and thus leads to a thorough understanding of the detailed structure of $\boldsymbol{G}$. Since the formalism of quantum conductance presented in this work uses a mixed basis set consisting of the plane waves and constriction states, the numerical results converge rapidly. For example, the conductance showing 7-8 steps can be handled with a reasonable accuracy by using $10 \times 10$ matrices for any $d$. Starting from the same type of basis set, the present formalism and that of Kirczenow ${ }^{9}$ have independently arrived at the similar expressions of $G$. The analogy with a $1 D$ potential problem is established in the present study, however. Moreover, our method is extended to study a more realistic situation, namely a nonuniform or smooth constriction geometry as described in Figs. 2 and 3, and the roughness of constriction as well. In this case the constriction is de- scribed by closely spaced uniform constrictions with different widths, and a transfer-matrix method is used for multiple boundary matching. The theory can also be extended to investigate various aspects such as temperature, scattering in the constriction, and magnetic field. While the effect of the temperature is being dealt with here, others will be reported subsequently.

Figure 1 illustrates the conductance $G\left(w / \lambda_{F}\right)$ calculated for the uniform constriction corresponding to $d=0, \lambda_{F}$, and $5 \lambda_{F}$ at $T=0 \mathrm{~K}$. The first curve $(d=0)$ corresponds to the Sharvin ${ }^{10}$ conductance, which was known to be $G_{s}=\left(2 e^{2} / h\right) 2 w / \lambda_{F}$. One sees that the whole curve is displaced, and weak oscillations are superimposed owing to the quantum-interference effects even at $d=0$. However, the longer $d$ is, the sharper the quantum jumps and the flatter the plateaus. Moreover, for $d \gtrsim 5 \lambda_{F}$ steps occur exactly at the integer multiples of $2 e^{2} / h$. The resonance structure, which originates from the interference of multiple reflected waves, becomes more pronounced at large $d$. Within the approximation of real and diagonal $\tilde{\mathbf{r}}$, the position of the $m$ th resonance on the $n$th plateau is estimated to be $w_{m n}=n \lambda_{F}\left[4-\left(m \lambda_{F} / d\right)^{2}\right]^{-1 / 2}$, and the number of resonances on the $n$th plateau $M_{n}=2 d / \lambda_{F}[(2 n+1) /$ $\left.(n+1)^{2}\right]^{1 / 2}$. Based on these approximate expressions we arrive at the conclusion that the number of resonances on 


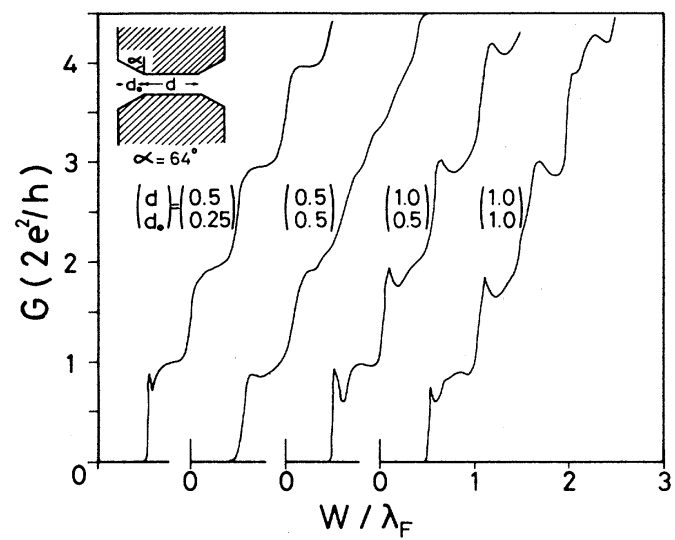

FIG. 3. Quantum conductance $G$ of a constriction with a smooth entrance calculated for various $\dot{d}_{0}$ and $d$ as described by the inset. The wedge angle of the smooth entrance is $\alpha=64^{\circ}$, and $T=0 \mathrm{~K}$.

a plateau is proportional to $d$, but decreases with increasing $w$. The same approximation also yields that $\delta G_{m n}$ (i.e., the difference in conductance between the $m$ th resonance and the next antiresonance on the $n$th plateau) decreases as either $m$ or $n$ increases. As for fixed $m$ and $n$, the larger $d$ is, the greater becomes $\delta G_{m n}$. Furthermore, we examined the effect of the intersubband mixing by neglecting the off-diagonal elements in $\tilde{\mathbf{K}}$ and found that $\delta G_{m n}$ is not affected in any essential manner. This is at variance with earlier results. ${ }^{6}$

In Fig. 2 we present the results obtained for the wedgelike constriction shown in the inset. $U p$ to a certain value of wedge-angle $\left(\alpha \sim 50^{\circ}\right)$, the conductance curve does not deviate from that of Sharvin contact geometry corresponding to $\alpha=0^{\circ}$, but beyond this value, the quantum steps become more pronounced. $\alpha=90^{\circ}$ corresponds to the uniform constriction shown in Fig. 1. At a given angle $\left(\alpha \gtrsim 60^{\circ}\right)$, the quantum effects are emphasized as $d$ increases. In spite of the apparent step structure at large $\alpha$, the resonance structure does not occur owing to the phase incoherence caused by the mixing among different subbands in the aperture. As an extension of this contact geometry we consider also the smooth entrance to a uniform constriction described in Fig. 3. The constriction geometry used by Khmelnitskii ${ }^{11}$ can be compared to the form described in Fig. 3 with $d_{0} / d \gg 1$ and $\alpha \approx 90^{\circ}$. Owing to the negligible band mixing and reflection in this geometry the quantization of conductance is not affected in any essential manner. However, because of insufficient phase coherence the resonance structure is weakened. In the present study the representation of a smooth tapering by a sequence of discrete steps may give rise to the reflections. These artificial reflections are, however, eliminated by using a large number of steps. It is found that the effect of the smooth entrance is insignificant for

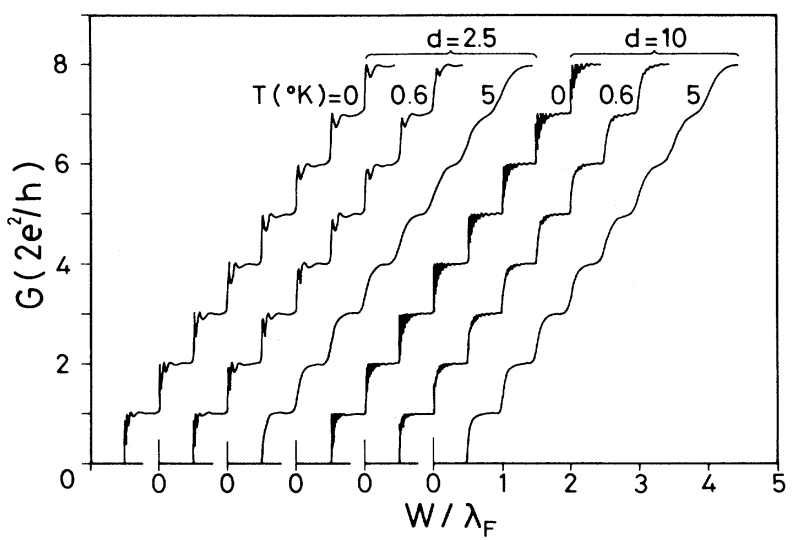

FIG. 4. Quantum conductance $G$ of uniform constriction of $d=2.5$ and 10 calculated for $T=0,0.6$, and $5 \mathrm{~K}$. The unit of length is $\lambda_{F}$, which is taken to' be $42 \mathrm{~nm}$ as in Ref. 4 .

$d_{0} / d \ll 1$ ( $d_{0}$ and $d$ are denoted by the inset), but becomes crucial if $d_{0} \sim d \sim \lambda_{F}$. In the latter case, additional structure is superimposed on the plateaus, and thus the step structure is deformed. Interestingly, the tight-binding calculations by Haanapel and van der $\mathrm{Marel}^{7}$ indicate that point imperfections in the constriction yield similar effects. Nonuniformities and roughness in the constriction deserve an extensive analysis, which will be reported subsequently.

Since carrier mobilities are decreased and the sharp Fermi distribution is smeared at finite but small temperatures, the quantum conductance is expected to deviate considerably from that at $T=0 \mathrm{~K}$. Ignoring the effects of inelastic scattering and assuming that $l_{e}$ is still larger than $d$, we studied the effect of temperature in the range from $T=0$ to $5 \mathrm{~K}$ within the ballistic transport regime. The quantum conductance $G\left(w / \lambda_{F}\right)$ was calculated for $T=0$, 0.6 , and $5 \mathrm{~K}$ and is shown in Fig. 4 . At $T=5 \mathrm{~K}$ the resonance structure completely disappeared. While the higher lying steps are smeared out, the steplike structure of the first ten channels are still maintained. In the range of temperature $(\$ 0.6 \mathrm{~K})$ where the experiments were performed, ${ }^{4,5}$ the resonance structure is maintained for small constriction length $\left(d \lesssim 2.5 \lambda_{F}\right)$. At small $d$ the resonance peaks are so widely separated that they persist in spite of the energy spreading due to temperature. In contrast to this, for long constrictions $\left(d \gtrsim 10 \lambda_{F}\right)$ closely spaced resonances can easily be destroyed. An important predict of this result is that the experimental data ${ }^{4,5}$ which do not display a clear resonance structure ought to be obtained from an effectively long constriction $(d \simeq 0.5 \mu \mathrm{m})$.

Part of this work has been carried out while one of the authors (E.T.) was visiting the International Center for Theoretical Physics, Trieste. We acknowledge valuable discussions with Professor A. Baratoff, Professor N. García, Professor C. Yalabik, and Dr. M. Büttiker. 
${ }^{1}$ R. Landauer, IBM J. Res. Dev. 1, 233 (1957); Z. Phys. B 68, 217 (1987).

${ }^{2}$ P. W. Anderson, D. J. Thouless, E. Abrahams, and D. S. Fisher, Phys. Rev. B 22, 3519 (1980).

${ }^{3}$ M. Büttiker, Phys. Rev. B 33, 3020 (1986).

${ }^{4}$ B. J. van Wees, H. van Houten, C. W. J. Beenakker, J. G. Williamson, L. P. Kouwenhoven, D. van der Marel, and C. T. Foxon, Phys. Rev. Lett. 60, 848 (1988).

${ }^{5}$ D. A. Wharam, T. J. Thornton, R. Newbury, M. Pepper, H.
Rithcie, and G. A. C. Jones, J. Phys. C 21, L209 (1988).

${ }^{6}$ A. D. Stone (unpublished).

${ }^{7}$ D. van der Marel (unpublished); E. G. Haanapel and D. van der Marel (unpublished).

${ }^{8}$ N. García (unpublished).

${ }^{9}$ G. Kirczenow, Solid State Commun. 68, 715 (1988).

${ }^{10}$ Yu. V. Sharvin, Zh. Eksp. Teor. Fiz. 48, 984 (1965) [Sov. Phys. JETP 21, 655 (1965)].

${ }^{11}$ D. E. Khmelnitskii (unpublished). 